\title{
Ultrasonic Investigation of Binary Solutions of Petrolium And Its Products
}

\author{
Deepak A. Zatale ${ }^{1}$, Sameer M. Bagade ${ }^{2}$, Ajay R. Chaware ${ }^{3}$ \\ ${ }^{1}$ Department of Physics, Govt. College of Engineering, Amravati, Maharashtra, India \\ 2 Department of Physics, Arts and Science College, Pulgaon, Maharashtra, India \\ ${ }^{3}$ Department of Physics, BD College of Engineering, Sevagram, Maharashtra, India
}

\begin{abstract}
Article Info

Volume 8, Issue 3

Page Number : 23-28

Publication Issue

May-June-2021

\section{Article History}

Accepted : 25 May 2021

Published : 30 May 2021

Experiment values of densities and ultrasonic speed of petroleum product Gasoline (Petrol) and 2T Oil were taken in different volume concentrations from 5\%, 10\%-----, and $95 \%$ at different temperatures from $298.15 \mathrm{~K}$ to $318.15 \mathrm{~K}$ having difference of $5 \mathrm{~K}$. From the experimental data, Apparent Molar Compressibility $\left(\phi_{K}\right)$, Relative Association $\left(R_{A}\right)$, Solvation Number $\left(S_{n}\right)$, Free Energy of Activation $(\Delta E)$, Excess Adiabatic Compressibility $\left(\beta_{a d}{ }^{E}\right)$, Excess Volume $\left(V^{E}\right)$, Excess Free Length $\left(L_{f}^{E}\right)$ have been computed. These parameters are used to focus light on the nature of component molecules of binary liquids and the excess functions are found to be sensitive to the nature and extent of the intermolecular interactions taking place in these binary mixtures.

Keywords: Ultrasonic velocity, Acoustical Parameters, Binary system, Molecular interactions.
\end{abstract}

\section{INTRODUCTION}

Knowledge of acoustic properties reveals the presence of molecular interactions between the component molecules in the multi-component liquid systems interaction plays an important role in the development of molecular sciences. ${ }^{[1-6]}$ Gasoline or petrol is a petroleum-derived liquid mixture consisting mostly of hydrocarbons and enhanced with benzene or iso-octane to increase octane ratings, used as fuel in internal combustion engines. For decades, Chevron Oronite has been a leader in the development of premium additive systems specifically designed to meet the unique lubrication demands of air-cooled, two-stroke cycle engines. In air-cooled applications, two-stroke cycle engines require an oil to provide reliable lubrication during high engine temperatures and under the most severe operating conditions. In continuation of our earlier work we have evaluated the acoustic Parameters, namely the Apparent Molar Compressibility $\left(\phi_{K}\right)$, Relative Association $\left(R_{A}\right)$, Solvation Number $\left(S_{n}\right)$, Free Energy of Activation $(\Delta E)$, Excess Adiabatic Compressibility $\left(\beta_{a d^{E}}\right)$, Excess Volume $\left(V^{E}\right)$, Excess Free Length $\left(L^{E}\right)$ for the binary mixtures Gasoline+ 2-T Oil. The results are discussed in terms of molecular interactions. ${ }^{[7-15]}$

\section{METHODS AND MATERIAL}

The ultrasonic velocities were measured at temperature at different temperatures and atmospheric pressure by using a single crystal variable 
path ultrasonic interferometer (F-81) operating at a frequency of $2 \mathrm{MHz}$. The temperature of the solution was maintained constant within $\pm 0.010 \mathrm{C}$ by circulation of water from thermostatically regulated water bath through the water- jacketed cell. The velocity measurements were precise to $\pm 0.5 \mathrm{~m} \mathrm{~s}-1$. Densities of the experimental liquids can also be measured by the hydrostatic plunger method, calibrated with deionised double distilled water with $0.9960 \times 103 \mathrm{~kg} \mathrm{~m}-3$ as its density at temperature $303.15 \mathrm{~K}$. The precision of density measurement was within $\pm 0.0003 \mathrm{~kg} \mathrm{~m}$-3. Different thermo-acoustical parameters such as apparent Molar Compressibility $\left(\phi_{K}\right)$, Relative Association $\left(R_{A}\right)$, Solvation Number $\left(S_{n}\right)$, Free Energy of Activation $(\Delta E)$, Excess Adiabatic Compressibility $\left(\beta_{a d^{E}}\right)$, Excess Volume $\left(V^{E}\right)$ and Excess Free Length $\left(L F^{E}\right)$ have been evaluated from the experimentally measured values of density, $\rho$ and ultrasonic velocity, $U^{[16-21]}$

$\mathrm{V}=\frac{M}{\rho}$

$\beta_{a d}=\frac{1}{u^{2} \rho}$

$L_{f}=K \times \beta^{1 / 2}$

$\Phi_{K}=\left(\rho_{0} \beta_{a d}-\rho \beta_{a d}^{0}\right) \times \frac{1000}{\rho_{0} C}+\frac{\beta_{a d}^{0} M_{2}}{\rho_{0}}$

$\mathrm{R}_{\mathrm{A}}=\left(\frac{\rho}{\rho_{0}}\right)\left(\frac{u_{0}}{u}\right)^{1 / 3}$

$S_{n}=\frac{n_{1}}{n_{2}}\left(1-\frac{\beta_{a d}}{\beta_{a d}^{0}}\right)$

$\Delta \mathrm{E}=$ Slope $\times \mathrm{R} \times 2.45$

$\beta_{a d}^{E}=\beta_{(E x p t)}-\beta_{(\text {Ideal })}$

$V_{a}^{E}=V_{(\text {Expt })}-V_{(\text {Ideeal })}$

$L_{f}^{E}=L_{f(\text { Expt })}-L_{f(\text { Ideal })}$

\section{RESULT AND DISCUSSION}

The apparent molar compressibility $\left(\phi_{K}\right)$ which decreased linearly with percentage volume concentration of mixtures at all five different temperatures have been shown in fig. 1.The positive value of $\phi_{K}$ shows strong electrostatic force in the vicinity of ion, causing electrostatic solution in ions. Fig.2 reveals the variation of relative association $\left(R_{A}\right)$ with percentage volume of mixture at five different temperatures which increased linearly. The increase in $R_{A}$ with concentration suggests that salvation of ions predominates over the breaking up of the solvent aggregates on addition substance. The variation of salvation number $\left(S_{n}\right)$ with percentage volume concentration of mixture at five all five temperatures exhibit in fig. 3 . The value $S_{n}$ decrease with increase in percentage volume and temperatures. The positive salvation number of solution suggests that the compressibility of the solution will be less than that of solvent.

The free energy of activation $(\Delta E)$ varies with percentage volume of mixtures shown in Fig. 4 . It has been found that $\Delta E$ increase nearly exponential as increase of percentage volume of mixture. Fig. 5 and 6 showed the variation of excess adiabatic compressibility and excess volume with percentage volume of mixture at five different temperatures. The figure show variation of $\beta_{a d^{E}}$ negative and $V^{E}$ positive with increase of percentage volume of mixture at all temperatures indicate an attractive interaction between two component liquid molecules in the mixture leading to an association between them. Fig.7 showed the variation of excess free length $L F$. The symmetrical positive variation of $L f^{E}$ at all temperatures supports attractive interaction. 
Table 1 : Apparent Molar Compressibility $\left(\phi_{K}\right) \mathrm{cm}^{3}$ $\mathrm{mol}^{-1}$

\begin{tabular}{|c|l|l|l|l|l|}
\hline $\mathbf{x}$ & $\begin{array}{l}298.15 \\
\%\end{array}$ & \multicolumn{1}{|c|}{$\begin{array}{l}303.15 \\
\mathbf{K}\end{array}$} & $\begin{array}{l}308.15 \\
\mathbf{K}\end{array}$ & $\begin{array}{l}313.15 \\
\mathbf{K}\end{array}$ & $\begin{array}{l}318.15 \\
\mathbf{K}\end{array}$ \\
\hline & $3.828 \mathrm{E}$ & $4.005 \mathrm{E}$ & $4.211 \mathrm{E}$ & $4.423 \mathrm{E}$ & $4.648 \mathrm{E}-$ \\
1 & -08 & -08 & -08 & -08 & 08 \\
\hline & $3.812 \mathrm{E}$ & $3.988 \mathrm{E}$ & $4.192 \mathrm{E}$ & $4.404 \mathrm{E}$ & $4.627 \mathrm{E}-$ \\
2 & -08 & -08 & -08 & -08 & 08 \\
\hline & $3.796 \mathrm{E}$ & $3.971 \mathrm{E}$ & $4.174 \mathrm{E}$ & $4.384 \mathrm{E}$ & $4.607 \mathrm{E}-$ \\
3 & -08 & -08 & -08 & -08 & 08 \\
\hline & $3.780 \mathrm{E}$ & $3.954 \mathrm{E}$ & $4.156 \mathrm{E}$ & $4.365 \mathrm{E}$ & $4.587 \mathrm{E}-$ \\
4 & -08 & -08 & -08 & -08 & 08 \\
\hline & $3.764 \mathrm{E}$ & $3.938 \mathrm{E}$ & $4.139 \mathrm{E}$ & $4.347 \mathrm{E}$ & $4.567 \mathrm{E}-$ \\
5 & -08 & -08 & -08 & -08 & 08 \\
\hline & $3.748 \mathrm{E}$ & $3.921 \mathrm{E}$ & $4.121 \mathrm{E}$ & $4.328 \mathrm{E}$ & $4.547 \mathrm{E}-$ \\
6 & -08 & -08 & -08 & -08 & 08 \\
\hline & $3.733 \mathrm{E}$ & $3.905 \mathrm{E}$ & $4.104 \mathrm{E}$ & $4.310 \mathrm{E}$ & $4.528 \mathrm{E}-$ \\
7 & -08 & -08 & -08 & -08 & 08 \\
\hline & $3.718 \mathrm{E}$ & $3.889 \mathrm{E}$ & $4.087 \mathrm{E}$ & $4.292 \mathrm{E}$ & $4.509 \mathrm{E}-$ \\
8 & -08 & -08 & -08 & -08 & 08 \\
\hline & $3.703 \mathrm{E}$ & $3.873 \mathrm{E}$ & $4.070 \mathrm{E}$ & $4.274 \mathrm{E}$ & $4.490 \mathrm{E}-$ \\
\hline 9 & -08 & -08 & -08 & -08 & 08 \\
\hline & $3.688 \mathrm{E}$ & $3.858 \mathrm{E}$ & $4.054 \mathrm{E}$ & $4.256 \mathrm{E}$ & $4.471 \mathrm{E}-$ \\
10 & -08 & -08 & -08 & -08 & 08 \\
\hline
\end{tabular}

\begin{tabular}{|l|l|l|l|l|l|} 
& 49 & 31 & 70 & 63 & 26 \\
\hline \multirow{3}{*}{8} & 1.0106 & 1.0103 & 1.0104 & 1.0100 & 1.0102 \\
& 07 & 78 & 96 & 70 & 16 \\
\hline \multirow{3}{*}{9} & 1.0117 & 1.0115 & 1.0116 & 1.0111 & 1.0113 \\
\hline \multirow{3}{*}{10} & 1.0129 & 24 & 21 & 76 & 05 \\
\hline
\end{tabular}

Table 3 : Solvation Number $\left(S_{n}\right)$

\begin{tabular}{|l|l|l|l|l|l|}
\hline $\begin{array}{r}\mathbf{x} \\
\mathbf{\%}\end{array}$ & $\mathbf{2 9 8 . 1 5 \mathrm { K }}$ & $\mathbf{3 0 3 . 1 5 \mathrm { K }}$ & $\mathbf{3 0 8 . 1 5 \mathrm { K }}$ & $313.15 \mathrm{~K}$ & $318.15 \mathrm{~K}$ \\
\hline & 0.33489 & 0.33433 & 0.34860 & 0.34363 & 0.35939 \\
1 & 6 & 1 & 6 & 9 & 7 \\
\hline & 0.29831 & 0.29925 & 0.30844 & 0.30793 & 0.31759 \\
2 & 4 & 6 & 0 & 0 & 1 \\
\hline & 0.28345 & 0.28486 & 0.29229 & 0.29321 & 0.30079 \\
3 & 6 & 6 & 6 & 8 & 6 \\
\hline & 0.27405 & 0.27567 & 0.28218 & 0.28378 & 0.29028 \\
4 & 5 & 7 & 7 & 7 & 5 \\
\hline & 0.26686 & 0.26859 & 0.27451 & 0.27649 & 0.28231 \\
5 & 1 & 0 & 5 & 1 & 2 \\
\hline & 0.26078 & 0.26257 & 0.26808 & 0.27028 & 0.27562 \\
6 & 6 & 2 & 1 & 3 & 9 \\
\hline & 0.25536 & 0.25718 & 0.26237 & 0.26471 & 0.26970 \\
7 & 8 & 1 & 0 & 4 & 0 \\
\hline & 0.25037 & 0.25219 & 0.25712 & 0.25955 & 0.26425 \\
8 & 2 & 5 & 5 & 7 & 6 \\
\hline & 0.24567 & 0.24749 & 0.25220 & 0.25468 & 0.25915 \\
9 & 0 & 1 & 3 & 8 & 0 \\
\hline & 0.24118 & 0.24299 & 0.24751 & 0.25003 & 0.25429 \\
10 & 3 & 4 & 8 & 2 & 1 \\
\hline
\end{tabular}

Table 2 : Relative Association $\left(R_{A}\right)$

\begin{tabular}{|c|c|c|c|c|c|}
\hline $\begin{array}{l}\mathbf{x} \\
\%\end{array}$ & $\begin{array}{c}298.15 \\
\text { K }\end{array}$ & $\begin{array}{c}303.15 \\
\mathrm{~K}\end{array}$ & $\begin{array}{c}308.15 \\
\mathrm{~K}\end{array}$ & $\begin{array}{c}313.15 \\
\mathrm{~K}\end{array}$ & $\begin{array}{c}318.15 \\
\mathrm{~K}\end{array}$ \\
\hline 1 & $\begin{array}{l}1.0024 \\
88\end{array}$ & $\begin{array}{l}1.0023 \\
43\end{array}$ & $\begin{array}{l}1.0026 \\
09\end{array}$ & $\begin{array}{l}1.0023 \\
15\end{array}$ & $\begin{array}{l}1.0025 \\
81\end{array}$ \\
\hline 2 & $\begin{array}{l}1.0036 \\
49\end{array}$ & $\begin{array}{l}1.0034 \\
92\end{array}$ & $\begin{array}{l}1.0037 \\
37\end{array}$ & $\begin{array}{l}1.0034 \\
23\end{array}$ & $\begin{array}{l}1.0036 \\
73\end{array}$ \\
\hline 3 & $\begin{array}{l}1.0048 \\
10\end{array}$ & $\begin{array}{l}1.0046 \\
41\end{array}$ & $\begin{array}{l}1.0048 \\
64\end{array}$ & $\begin{array}{l}1.0045 \\
32\end{array}$ & $\begin{array}{l}1.0047 \\
64\end{array}$ \\
\hline 4 & $\begin{array}{l}1.0059 \\
71\end{array}$ & $\begin{array}{l}1.0057 \\
89\end{array}$ & $\begin{array}{l}1.0059 \\
91\end{array}$ & $\begin{array}{l}1.0056 \\
40\end{array}$ & $\begin{array}{l}1.0058 \\
55\end{array}$ \\
\hline 5 & $\begin{array}{l}1.0071 \\
31\end{array}$ & $\begin{array}{l}1.0069 \\
37\end{array}$ & $\begin{array}{l}1.0071 \\
18\end{array}$ & $\begin{array}{l}1.0067 \\
48\end{array}$ & $\begin{array}{l}1.0069 \\
45\end{array}$ \\
\hline 6 & $\begin{array}{l}1.0082 \\
90\end{array}$ & $\begin{array}{l}1.0080 \\
84\end{array}$ & $\begin{array}{l}1.0082 \\
44\end{array}$ & $\begin{array}{l}1.0078 \\
55\end{array}$ & $\begin{array}{l}1.0080 \\
36\end{array}$ \\
\hline 7 & 1.0094 & 1.0092 & 1.0093 & 1.0089 & 1.0091 \\
\hline
\end{tabular}

Table 4 : Free Energy of Activation $(\Delta E) \mathrm{J} \mathrm{mol}^{-1}$ $\mathbf{x} \% \quad \Delta \mathrm{E}$

$1 \quad 7387.734$

$2 \quad 7422.859$

\begin{tabular}{l|l}
3 & 7455.061
\end{tabular}

\begin{tabular}{l|l}
4 & 7484.692
\end{tabular}

$5 \quad 7512.041$

$6 \quad 7537.370$

\begin{tabular}{l|l}
7 & 7560.890
\end{tabular}

$8 \quad 7582.790$ 


\section{$9 \quad 7603.232$ \\ \begin{tabular}{l|l}
10 & 7622.358
\end{tabular}}

Table 5 : Excess Adiabatic Compressibility $\left(\beta_{a d^{E}}\right) \mathrm{cm}^{2}$ dyne $^{-1}$

\begin{tabular}{|c|c|c|c|c|c|}
\hline $\mathrm{x} \%$ & $298.15 K$ & $303.15 \mathrm{~K}$ & $308.15 K$ & $313.15 \mathrm{~K}$ & $318.15 \mathrm{~K}$ \\
\hline 1 & $\begin{array}{l}-4.77 \mathrm{E}- \\
13\end{array}$ & $\begin{array}{l}-4.91 \mathrm{E}- \\
13\end{array}$ & $\begin{array}{l}-5.54 \mathrm{E}- \\
13\end{array}$ & $\begin{array}{l}-5.56 \mathrm{E}- \\
13\end{array}$ & $\begin{array}{l}-6.32 \mathrm{E}- \\
13\end{array}$ \\
\hline 2 & $\begin{array}{l}-7.51 \mathrm{E}- \\
13\end{array}$ & $\begin{array}{l}-7.81 \mathrm{E}- \\
13\end{array}$ & $\begin{array}{l}-8.65 \mathrm{E}- \\
13\end{array}$ & $\begin{array}{l}-8.89 \mathrm{E}- \\
13\end{array}$ & $\begin{array}{l}-9.88 \mathrm{E}- \\
13\end{array}$ \\
\hline 3 & $\begin{array}{l}-1.02 \mathrm{E}- \\
12\end{array}$ & $\begin{array}{l}-1.06 \mathrm{E}- \\
12\end{array}$ & $\begin{array}{l}-1.17 \mathrm{E}- \\
12\end{array}$ & $\begin{array}{l}-1.21 \mathrm{E}- \\
12\end{array}$ & $\begin{array}{l}-1.33 \mathrm{E}- \\
12\end{array}$ \\
\hline 4 & $\begin{array}{l}-1.28 \mathrm{E}- \\
12\end{array}$ & $\begin{array}{l}-1.33 \mathrm{E}- \\
12\end{array}$ & $\begin{array}{l}-1.46 \mathrm{E}- \\
12\end{array}$ & $\begin{array}{l}-1.53 \mathrm{E}- \\
12\end{array}$ & $\begin{array}{l}-1.67 \mathrm{E}- \\
12\end{array}$ \\
\hline 5 & $\begin{array}{l}-1.53 \mathrm{E}- \\
12\end{array}$ & $\begin{array}{l}-1.60 \mathrm{E}- \\
12\end{array}$ & $\begin{array}{l}-1.74 \mathrm{E}- \\
12\end{array}$ & $\begin{array}{l}-1.83 \mathrm{E}- \\
12\end{array}$ & $\begin{array}{l}-1.99 \mathrm{E}- \\
12\end{array}$ \\
\hline 6 & $\begin{array}{l}-1.77 \mathrm{E}- \\
12\end{array}$ & $\begin{array}{l}-1.86 \mathrm{E}- \\
12\end{array}$ & $\begin{array}{l}-2.02 \mathrm{E}- \\
12\end{array}$ & $\begin{array}{l}-2.12 \mathrm{E}- \\
12\end{array}$ & $\begin{array}{l}-2.31 \mathrm{E}- \\
12\end{array}$ \\
\hline 7 & $\begin{array}{l}-2.00 \mathrm{E}- \\
12\end{array}$ & $\begin{array}{l}-2.10 \mathrm{E}- \\
12\end{array}$ & $\begin{array}{l}-2.28 \mathrm{E}- \\
12\end{array}$ & $\begin{array}{l}-2.41 \mathrm{E}- \\
12\end{array}$ & $\begin{array}{l}-2.61 \mathrm{E}- \\
12\end{array}$ \\
\hline 8 & $\begin{array}{l}-2.23 \mathrm{E}- \\
12\end{array}$ & $\begin{array}{l}-2.34 \mathrm{E}- \\
12\end{array}$ & $\begin{array}{l}-2.54 \mathrm{E}- \\
12\end{array}$ & $\begin{array}{l}-2.68 \mathrm{E}- \\
12\end{array}$ & $\begin{array}{l}-2.91 \mathrm{E}- \\
12\end{array}$ \\
\hline 9 & $\begin{array}{l}-2.45 \mathrm{E}- \\
12\end{array}$ & $\begin{array}{l}-2.58 \mathrm{E}- \\
12\end{array}$ & $\begin{array}{l}-2.79 \mathrm{E}- \\
12\end{array}$ & $\begin{array}{l}-2.95 \mathrm{E}- \\
12\end{array}$ & $\begin{array}{l}-3.19 \mathrm{E}- \\
12\end{array}$ \\
\hline 10 & $\begin{array}{l}-2.66 \mathrm{E}- \\
12\end{array}$ & $\begin{array}{l}-2.80 \mathrm{E}- \\
12\end{array}$ & $\begin{array}{l}-3.03 \mathrm{E}- \\
12\end{array}$ & $\begin{array}{l}-3.21 \mathrm{E}- \\
12\end{array}$ & $\begin{array}{l}-3.47 \mathrm{E}- \\
12\end{array}$ \\
\hline
\end{tabular}

Table 6 : Excess Volume $\left(V^{E}\right) \mathrm{cm}^{3} \mathrm{~mol}^{-1}$

\begin{tabular}{|c|c|c|c|c|c|}
\hline $\mathrm{x} \%$ & $298.15 K$ & $303.15 \mathrm{~K}$ & $308.15 \mathrm{~K}$ & $313.15 \mathrm{~K}$ & $318.15 \mathrm{~K}$ \\
\hline 1 & $1.008 \mathrm{E}+0$ & $1.028 \mathrm{E}+00$ & $9.921 \mathrm{E}-01$ & $1.038 \mathrm{E}+00$ & $\begin{array}{l}9.978 \mathrm{E}- \\
01\end{array}$ \\
\hline 2 & $2.165 \mathrm{E}+0$ & $2.193 \mathrm{E}+00$ & $2.163 \mathrm{E}+00$ & $2.215 \mathrm{E}+00$ & $2.181 \mathrm{E}+0$ \\
\hline 3 & $3.256 \mathrm{E}+0$ & $3.290 \mathrm{E}+00$ & $3.266 \mathrm{E}+00$ & $3.324 \mathrm{E}+00$ & $3.296 \mathrm{E}+0$ \\
\hline 4 & $4.284 \mathrm{E}+0$ & $4.325 \mathrm{E}+00$ & $4.306 \mathrm{E}+00$ & $4.370 \mathrm{E}+00$ & $4.347 \mathrm{E}+0$ \\
\hline 5 & $5.252 \mathrm{E}+0$ & $5.299 \mathrm{E}+00$ & $5.285 \mathrm{E}+00$ & $5.354 \mathrm{E}+00$ & $5.337 \mathrm{E}+0$ \\
\hline 6 & $6.164 \mathrm{E}+0$ & $6.215 \mathrm{E}+00$ & $6.206 \mathrm{E}+00$ & $6.281 \mathrm{E}+00$ & $6.268 \mathrm{E}+0$ \\
\hline 7 & $7.021 \mathrm{E}+0$ & $7.077 \mathrm{E}+00$ & 7.073E+00 & $7.152 \mathrm{E}+00$ & $7.144 \mathrm{E}+0$ \\
\hline 8 & $7.826 \mathrm{E}+0$ & $7.888 \mathrm{E}+00$ & $7.888 \mathrm{E}+00$ & $7.971 \mathrm{E}+00$ & $7.967 \mathrm{E}+0$ \\
\hline 9 & $8.582 \mathrm{E}+0$ & $8.648 \mathrm{E}+00$ & $8.652 \mathrm{E}+00$ & $8.740 \mathrm{E}+00$ & $8.740 \mathrm{E}+0$ \\
\hline 10 & $9.291 \mathrm{E}+0$ & $9.362 \mathrm{E}+00$ & $9.370 \mathrm{E}+00$ & $9.462 \mathrm{E}+00$ & $9.465 \mathrm{E}+0$ \\
\hline
\end{tabular}

Table 7 : Excess Free Length $\left(L f^{E}\right) \mathrm{cm}$

\begin{tabular}{|l|l|l|l|l|l|}
\hline $\begin{array}{l}\mathbf{x} \\
\%\end{array}$ & $298.15 \mathrm{~K}$ & $303.15 \mathrm{~K}$ & $308.15 \mathrm{~K}$ & $313.15 \mathrm{~K}$ & $318.15 \mathrm{~K}$ \\
\hline & $1.907 \mathrm{E}-$ & $2.109 \mathrm{E}-$ & $2.023 \mathrm{E}-$ & $1.949 \mathrm{E}-$ & $1.650 \mathrm{E}-$ \\
1 & 11 & 11 & 11 & 11 & 11 \\
\hline & $4.229 \mathrm{E}-$ & $4.529 \mathrm{E}-$ & $4.522 \mathrm{E}-$ & $4.565 \mathrm{E}-$ & $4.350 \mathrm{E}-$ \\
2 & 11 & 11 & 11 & 11 & 11 \\
\hline & $6.412 \mathrm{E}-$ & $6.804 \mathrm{E}-$ & $6.872 \mathrm{E}-$ & $7.025 \mathrm{E}-$ & $6.888 \mathrm{E}-$ \\
3 & 11 & 11 & 11 & 11 & 11 \\
\hline & $8.464 \mathrm{E}-$ & $8.943 \mathrm{E}-$ & $9.080 \mathrm{E}-$ & $9.337 \mathrm{E}-$ & $9.272 \mathrm{E}-$ \\
4 & 11 & 11 & 11 & 11 & 11 \\
\hline & $1.039 \mathrm{E}-$ & $1.095 \mathrm{E}-$ & $1.115 \mathrm{E}-$ & $1.151 \mathrm{E}-$ & $1.151 \mathrm{E}-$ \\
5 & 10 & 10 & 10 & 10 & 10 \\
\hline & $1.220 \mathrm{E}-$ & $1.284 \mathrm{E}-$ & $1.310 \mathrm{E}-$ & $1.355 \mathrm{E}-$ & $1.361 \mathrm{E}-$ \\
6 & 10 & 10 & 10 & 10 & 10 \\
\hline & $1.390 \mathrm{E}-$ & $1.461 \mathrm{E}-$ & $1.492 \mathrm{E}-$ & $1.546 \mathrm{E}-$ & $1.558 \mathrm{E}-$ \\
7 & 10 & 10 & 10 & 10 & 10 \\
\hline & $1.549 \mathrm{E}-$ & $1.627 \mathrm{E}-$ & $1.663 \mathrm{E}-$ & $1.725 \mathrm{E}-$ & $1.743 \mathrm{E}-$ \\
8 & 10 & 10 & 10 & 10 & 10 \\
\hline & $1.697 \mathrm{E}-$ & $1.782 \mathrm{E}-$ & $1.823 \mathrm{E}-$ & $1.893 \mathrm{E}-$ & $1.915 \mathrm{E}-$ \\
9 & 10 & 10 & 10 & 10 & 10 \\
\hline & $1.837 \mathrm{E}-$ & $1.927 \mathrm{E}-$ & $1.973 \mathrm{E}-$ & $2.050 \mathrm{E}-$ & $2.077 \mathrm{E}-$ \\
10 & 10 & 10 & 10 & 10 & 10 \\
\hline
\end{tabular}

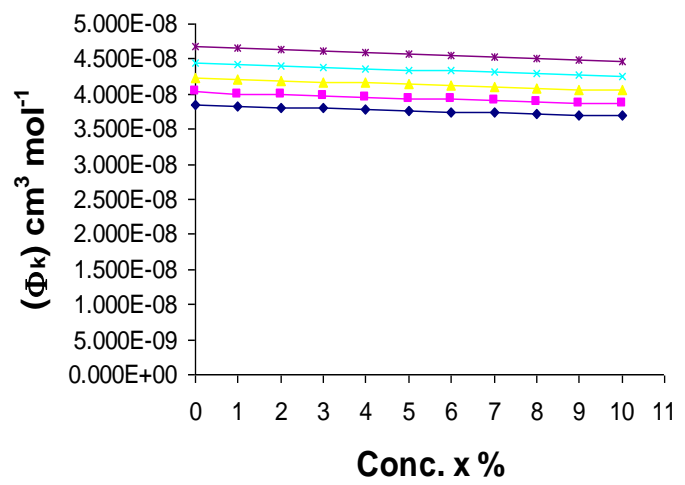

Figure 1 : Volume conc. $\mathrm{x} \%$ versus Apparent Molar Compressibility $\left(\phi_{K}\right)$

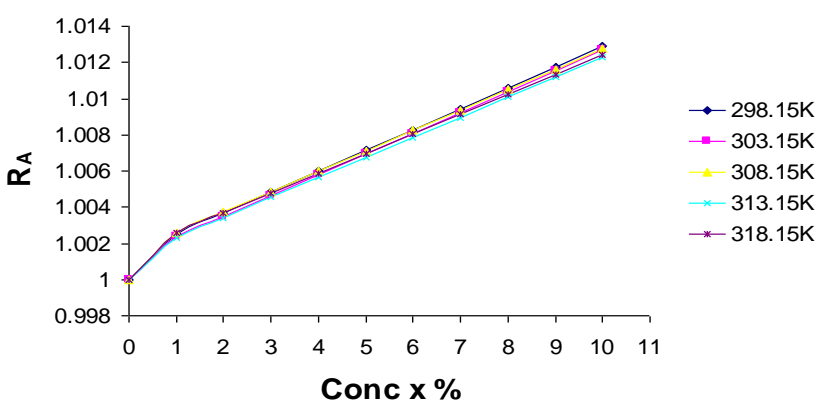

Figure 2 : Volume conc. $\mathrm{x} \%$ versus Relative Association $\left(R_{A}\right)$ 


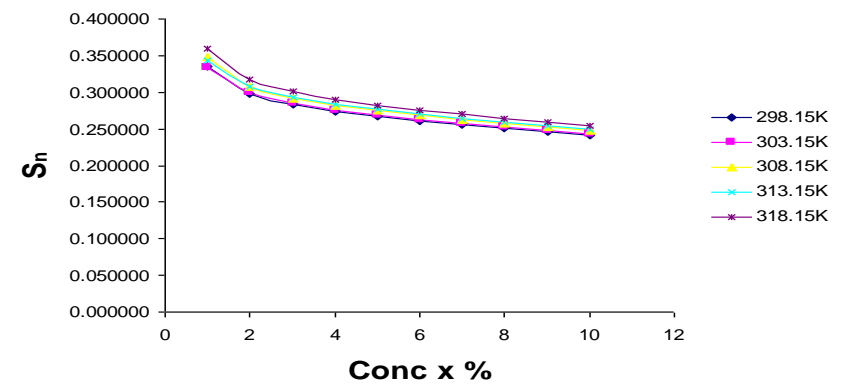

Figure 3 : Volume conc. $\mathrm{x} \%$ versus Solvation Number $\left(S_{n}\right)$

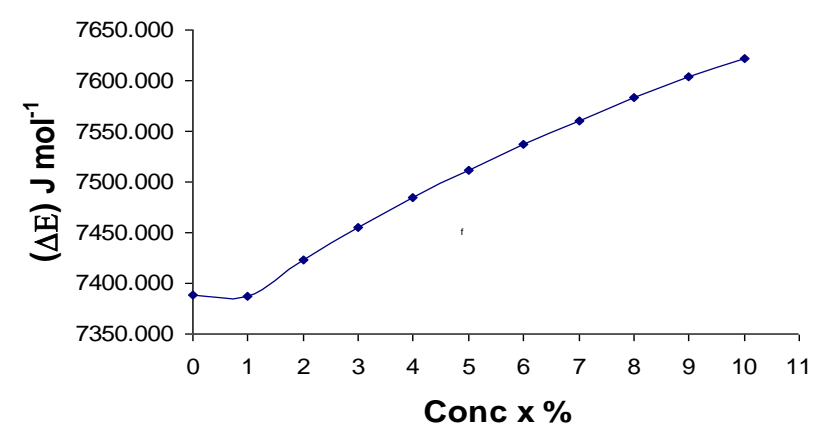

Figure 4 : Volume conc. $\mathrm{x} \%$ versus Free Energy of Activation $(\Delta E)$

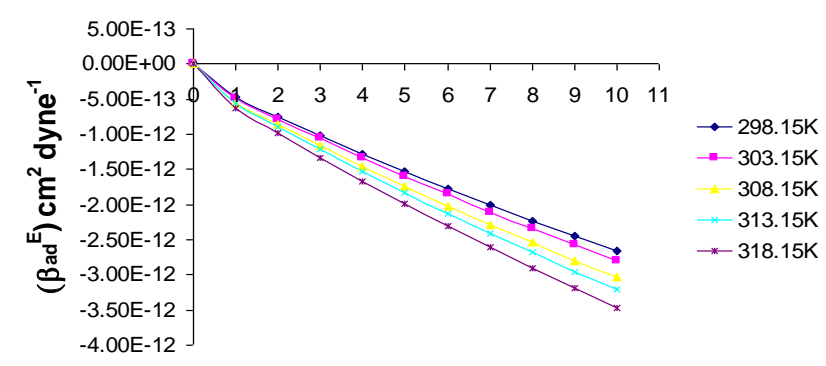

Conc $x \%$

Figure 5: Volume conc. $\mathrm{x} \%$ versus $\beta_{a d}{ }^{E}$

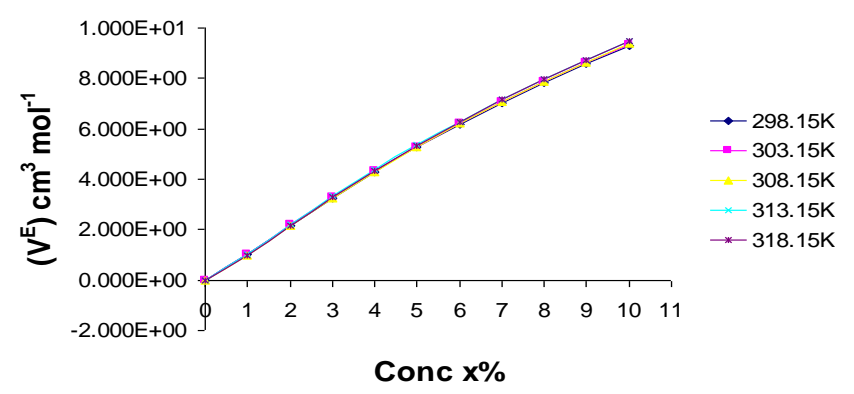

Figure 6: Volume conc. $\mathrm{x} \%$ versus $V^{E}$

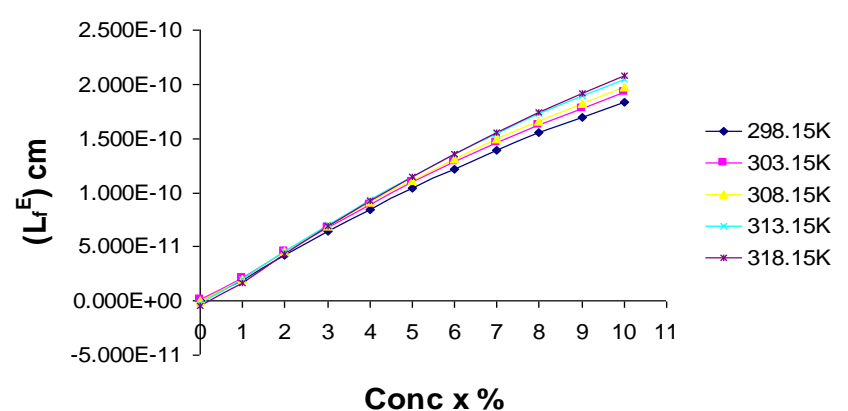

Figure 7 : Volume conc. $\mathrm{x} \%$ versus $L f^{E}$

\section{CONCLUSION}

The 2T Oil (fuel oil's) which we used are non polar solvents and miscible in gasoline and there are weak interaction unto lower level of \% concentration, the negative value of $V^{E}$ show that the molecules set free from the original cluster and rate of broken of cluster depends on nature of $\beta_{a d}{ }^{E}$ and $V^{E}$. Free Length Theory works not so well when applied to mixtures.

\section{REFERENCES}

[1]. Hobbs, M.E. and Bates, W.W., J.Am. Chem. Soc., 74,746, (1952).

[2]. Negakuva., J.Am. Chem. Soc., 76,3070, (1954).

[3]. Freedman, E., J.Chem. Phys., 21, 1784, (1955).

[4]. Kannappan, A.N. and Rajendran, V., Indian J.Pure and Appl. Phys., 30,176, (1992).

[5]. Hyderkhan, V.and Subramanyam, S.V., Tras. Parad Soc. (GB) 67,2282, (1971).

[6]. Temperley, H.N.V., Rawlinson, J.S. and Rush brooke, G.S., Phys. of simple liquids (John wiley,Newyork), (1968).

[7]. Glasstone, S., Laidler, K.J., and Erying, H., Theory of Rate Processes.

[8]. Mc. Graw - Hill, Newyork, 478, 479, (1950). Erying, H.and Kincaid, J.F.J. Chem. Phy., 6,520,(1938).

[9]. Pino, Paulina Environmental Health, (2004)

[10]. Jacobson B. Acta Chem. Scand, 5 (1951) 1214.

[11]. Jacobson B. Acta Chem. Scand, 6 (1952) 1485.

[12]. Kitter C. J. J. Chem. Phys., 14 (1946) 64. 
[13]. Schaaffs W. Z. Phys., 144 (1939) 100.

[14]. Schaaffs W. Ann-Phys. Lpz., 40 (1941) 393.

[15]. H. Reiss, H. L. Frisch and J. L. Lebowitz J. Chem. Phys., 31 (1959) 369.

[16]. H. Reiss, H. L. Frisch, E. Helfand and J. L. Lebowitzidbi., 32 (1960) 119.

[17]. H. Reiss, H. L. Frisch and J. L. Lebowitz idbi., 33 (1979) 1379.

[18]. H. Reiss, H. L. Frisch and J. L. Lebowitz idbi., 34 (1961) 1037

[19]. M. R. Rao Ind. J. Phys., 14 (1940) 109.

[20]. M. R. Rao J. Chem. Phys., 14 (1941) 682.

[21]. Nomoto O. J. Phys. Soc. Japan, 8 (1983) 553.

\section{Cite this article as :}

Deepak A. Zatale, Sameer M. Bagade, Ajay R. Chaware, "Ultrasonic Investigation of Binary Solutions of Petrolium And Its Products", International Journal of Scientific Research in Science and Technology (IJSRST), Online ISSN : 2395-602X, Print ISSN : 2395-6011, Volume 8 Issue 3, pp. 23-28, May-June 2021. Available at doi : https://doi.org/10.32628/IJSRST218310

Journal URL : https://ijsrst.com/IJSRST218310 